Pacific Journal of Mathematics

CAPACITY THEORY IN BANACH SPACES 


\title{
CAPACITY THEORY IN BANACH SPACES
}

\author{
Peter A. Fowler
}

In classical potential theory one way of defining capacity of a compact $K \subset R^{n}$ puts cap $K$ equal to the total mass of $\mu$, where $\mu$ is the measure associated with the inferior envelope of the family of nonnegative superharmonic functions majorizing the characteristic function $I_{K}$. A second (equivalent) definition puts cap $K=1 /\left\|\gamma_{0}\right\|_{e}$ where $\gamma_{0}$ is the projection of the null measure onto the set of positive Radon measures $\gamma$ supported by $K$, satisfying $\int d r \geqq 1$ and having finite energy: $\|\gamma\|_{e}=\int U^{r} d r<+\infty$.

In the axiomatic Hilbert space setting of Dirichlet spaces Beurling and Deny have shown that equivalence of definitions of the two above types leads to a rich capacity theory. In this article all of these results are extended to the family of Banach-Dirichlet (BD) spaces, i.e., uniformly convex Banach spaces of (equivalence classes of) functions satisfying the Dirichlet space axioms. This is accomplished by using a capacity of the first type in the $\mathrm{BD}$ space $D$, and of the second type in the dual space $D^{\prime}$.

THEOREM 1. The two types of capacity are equal.

THEOREM 2. Exterior capacity is a true capacity in the sense of Brelot.

THEOREM 3. A set $E$ has zero exterior capacity iff $E$ is capacitable and $\mu E=0$ for all measures $\mu$ generating a pure potential $u^{\mu} \in D^{\prime}$.

THEOREM 4. For every quasi-continuous representative $u^{*}$ of $u \in D$ and every $\mu$ generating a pure potential $u^{\mu}$, the formula $\left(u, u^{\mu}\right)=\int u^{*} d \mu$ holds, where $(\cdot, \cdot)$ is the bi-linear form on $D \times D^{\prime}$.

The reader will be aided by familiarity with [6]. Some definitions therein will be reiterated in $\S 2$.

1. Preliminary lemmas concerning certain Banach spaces, Let $\left\{E_{i}\right\}_{i \in I}$ be a nonvoid family of nonvoid subsets of a set $A$.

DeFINITION 1.1. The family $\left\{E_{i}\right\}_{i \in I}$ is directed downward by inclusion if for each pair $i, j \in I$ there exists $k \in I$ with $E_{k} \subset E_{i} \cap E_{j}$.

The family $\left\{E_{i}\right\}$ is also called a filter base.

DeFINITION 1.2. The family $\left\{E_{i}\right\}_{i \in I}$ is directed upward by containment if for each pair $i, j \in I$ there exists $k \in I$ with $E_{k} \supset E_{i} \cup E_{j}$. 
Note. If $\left\{E_{i}\right\}_{i \in I}$ is directed downward by inclusion (upward by containment) and $x_{i} \in E_{i}$ for each $i \in I$, then $\left\{x_{i}\right\}_{i \in I}$ is a net in $A$ when $I$ is directed by the rule $i \geqq j$ iff $E_{i} \subset E_{j}\left(E_{i} \supset E_{j}\right)$.

Lemma 1.3. In a Banach space $B$ with norm $\|\cdot\|$ let $\left\{E_{i}\right\}_{i \in I}$ be a family of closed convex sets directed downward by inclusion such that the set of numbers $\left\{\inf \left\{\|\boldsymbol{z}\| \mid \boldsymbol{z} \in E_{i}\right\}\right\}_{i \in I}$ has a supremum $M<\infty$.

(i) If $B$ is reflexive, then $E=\bigcap_{i \in I} E_{i} \neq \varnothing$ and there exists $z \in E$ with $\|\boldsymbol{z}\| \leqq M$.

(ii) If $B$ is uniformly convex and for each $i \in I, x_{i}$ is the unique element of minimum norm of $E_{i}$, then the net $\left\{x_{i}\right\}_{i \in I}$ is Cauchy and $x=\lim _{i \in I} x_{i}$ is the unique element of minimum norm of $E$.

Proof. (i) Let $B_{M}=\{z \in B\|\| z \| \leqq M\}$. The family $\left\{E_{i} \cap B_{M}\right\}_{i \in I}$ is directed downward by inclusion. Each $E_{i} \cap B_{M}$ is closed and convex, thus weakly closed. Since $B$ is reflexive, $B_{M}$ is weakly compact. Hence $\bigcap_{i \in I} E_{i} \cap B_{M} \neq \varnothing$, i.e., there exists $z \in E$ with $\|z\| \leqq M$.

(ii) Since each $E_{i}$ is closed and convex, $E$ is also. By uniform convexity there exists a unique $x \in E$ of minimum norm and (i) assures $\|x\| \leqq M$. Moreover, $x \in E_{i}$ for each $i \in I$ and $M=\sup \left\{\left\|x_{i}\right\| \mid i \in I\right\}$ entail $\|x\| \geqq M$. Thus $\|x\|=M$.

The net $\left\{x_{i}\right\}_{i \in I}$ is Cauchy. In fact, it is clear that $\lim _{i \in I}\left\|x_{i}\right\|=M$, i.e., for $\varepsilon>0$ there exists $i \in I$ such that $j \geqq i$ implies $\left\|x_{j}\right\|>M-\varepsilon / 2$. Moreover, for all $j, k \geqq i$,

$$
2 M \geqq\left\|x_{k}\right\|+\left\|x_{j}\right\| \geqq\left\|x_{k}+x_{j}\right\|=2\left\|\left(x_{k}+x_{j}\right) / 2\right\| \text {. }
$$

But $x_{k}, x_{j} \in E_{i}$ so convexity assures $\left(x_{k}+x_{j}\right) / 2 \in E_{i}$. Since $x_{i}$ is the unique element of minimum norm in $E_{i}$, we have

$$
2 M \geqq 2\left\|\left(x_{k}+x_{j}\right) / 2\right\| \geqq 2\left\|x_{i}\right\|>2 M-\varepsilon .
$$

This shows $\lim _{j, k \in I}\left\|x_{k}+x_{j}\right\|=2 M$. The fact that $\left\{x_{i}\right\}_{i \in I}$ is Cauchy follows directly from the definition of uniform convexity. Put $y=$ $\lim x_{i}$. Then $\|y\|=\lim \left\|x_{i}\right\|=M$. Since $\left\{E_{i}\right\}_{i \in I}$ is directed downward by inclusion and each $E_{i}$ is closed, we have $y \in \bigcap_{i \in I} E_{i}=E$. But $x$ is the unique element of minimum norm in $E$, so $y=x$.

LEMMA 1.4. Let $B$ be a uniformly convex Banach space and $\left\{E_{i}\right\}_{i \in I}$ a family of closed convex subsets of $B$ directed upward by containment. Let $K \subset B$ be closed and convex with $K \supset \bigcup_{i \in I} E_{i}$. Denote by $x_{i}, x$ the unique elements of minimum norm of $E_{i}, K$ respectively. If $\|x\|=\inf \left\{\left\|x_{i}\right\| \mid i \in I\right\}$, then $\lim _{i \in I} x_{i}=x$.

Proof. To see that $\left\{x_{i}\right\}_{i \in I}$ is Cauchy, first observe that $\lim \left\|x_{i}\right\|=$ 
$\inf \left\{\left\|x_{i}\right\| \mid i \in I\right\}=\|x\|$. Given $\varepsilon>0$ choose $n \in I$ such that $m \geqq n$ implies $\|x\|+\varepsilon / 2 \geqq\left\|x_{m}\right\|$. Then for $i, j \geqq n$,

$$
\begin{aligned}
& 2\|x\|+\varepsilon \geqq\left\|x_{i}\right\|+\left\|x_{j}\right\| \geqq\left\|x_{i}+x_{j}\right\| \\
= & 2\left\|\left(x_{i}+x_{j}\right) / 2\right\| \geqq 2\left\|x_{m}\right\|
\end{aligned}
$$

for any $m \geqq i, j$ since $m \geqq i, j$ implies $E_{m} \supset E_{i} \cup E_{j}$ and $E_{m}$ is convex. Thus $\lim _{i, j \in I}\left\|x_{i}+x_{j}\right\|=2\|x\|$ and uniform convexity assure $\left\{x_{i}\right\}$ is Cauchy. Put $y=\lim x_{i}$. As in the proof of Lemma 1.3, $y=x$.

COROLlaRY 1.5. Let $B,\left\{E_{i}\right\}_{i \in I},\left\{x_{i}\right\}_{i \in I}$ be as in Lemma 1.4. Then $H=\overline{\mathrm{U} E_{i}}$ is the closed convex hull of $\bigcup E_{i}$, and $\lim x_{i}=x$ where 2 denotes the unique element of minimum norm in $H$.

Proof. Since each $E_{i}$ is convex and family is directed upward by containment, $\bigcup E_{i}$ is convex. Thus $\mathrm{H}=\overline{\mathrm{U} E_{i}}$ is the closed convex hull of $\bigcup E_{i}$. Given $\varepsilon>0$ there exists $i \in I$ and $z \in E_{i}$ with $\|x\| \geqq$ $\|\boldsymbol{z}\|-\varepsilon \geqq\left\|x_{i}\right\|-\varepsilon$, so $\|x\|=\inf \left\{\left\|x_{i}\right\| \mid i \in I\right\}$ and Lemma 1.4 applies.

2. Review of definitions and basic facts. Much of the below is expanded upon in [6].

A normal contraction $T: R \rightarrow R$ of the line verifies $T(0)=0$ and $|T(x)-T(y)| \leqq|x-y|$. A duality map $S: N \rightarrow N^{\prime}$ of a smooth normed linear space $N$ to its dual is the unique map satisfying $\|S(u)\|=$ $\|u\|$ and $|(u, S(u))|=\|u\|^{2}$. Also, for nonzero $u \in N$

$$
(x, S(u))=\|u\| \cdot \lim _{t \rightarrow o} \frac{\|u+t x\|-\|u\|}{t}
$$

for all $x \in N$. Let $X$ denote a locally compact Hausdorff space, $\mathscr{C}=\mathscr{C}(X)$ the continuous real valued functions $\varphi$ on $X$ with support $\mathscr{S}(\varphi)$ compact supplied with the inductive limit topology, $\xi$ a positive Radon measure on $X$. Let $F=F(X, \xi)$ denote a Banach space with norm $\|\cdot\|$ of equivalence classes of real valued, locally $\xi$-integrable functions on $X$. As with $L^{p}$ spaces, we assume each equivalence class contains all functions which are equal $\xi$-a.e. to a given representative of that class. (A departure from this convention is suggested in $\S 10$, where "refinements" of classes are considered.)

The three Dirichlet axioms are

(a) For any compact $K \subset X$ there exists a constant $A(K) \geqq 0$ such that for $u \in F$

$$
\int_{K}|u| d \xi \leqq A(K)\|u\|
$$

(b) The measure $\xi$ is everywhere dense in $X$, and $F \cap \mathscr{C}$ is dense in $F$ and in $\mathscr{C}$. 
(c) For any normal contraction $T$ and $u \in F$ we have the composition $T u \in F$ and $\|T u\| \leqq\|u\|$.

A Banach-Dirichlet (BD) space is a Banach space $D=D(X, \xi)$ of equivalence classes of real valued locally $\xi$-integrable functions which satisfies the three Dirichlet axioms. Several examples of BD spaces are given in [6]. Pure potentials are elements of the positive dual cone $F^{\prime+}$ where the natural order is assumed on $F$. If $F$ is uniformly convex and satisfies axioms (a) and (c), then $S(u) \in F^{\prime+}$ implies $u \geqq 0$ a.e. $\xi$. If $f \in D^{\prime+}$ where $D$ is a $\mathrm{BD}$ space, there exists a unique Radon measure $\mu \geqq 0$ such that

$$
(\varphi, f)=\int \varphi d \mu \text { for all } \varphi \in D \cap \mathscr{C} \text {. }
$$

The measure associated with $f$ is $\mu$ and $\mu$ generates $f$. Write $f=u^{\mu}$, or in case $\mu=g \cdot \xi$, write $f=u^{g}$. A potential $f$ satisfies (1) where $\mu$ need not be positive.

3. Capacity and dual capacity of open sets. Throughout the remainder of this article it is assumed that $F(X, \xi)$ is uniformly convex and verifies axiom (a).

DEFinition 3.1. Let $\omega \subset X$ be an open set.

(i) $\mathscr{U}_{\omega} \subset F$ is defined

$$
\mathscr{U}_{\omega}=\{u \in F \mid u \geqq 1 \text { a.e. } \xi \text { on } \omega\} \text {. }
$$

(ii) The capacity of $\omega$ is a nonnegative real number or $+\infty$ given by

$$
\operatorname{cap} \omega=\inf \left\{\|u\| \mid u \in \mathscr{C}_{\omega}\right\} .
$$

(iii) If $\mathscr{K}_{\omega} \neq \varnothing$, the unique element of minimum norm of $\mathscr{K}_{\omega}$ is called the capacitary element associated with $\omega$.

Using axiom (a) it is easy to show $\mathscr{K}_{\omega}$ is closed and convex, thus (iii) follows. In case $\mathscr{U}_{\omega}=\varnothing$, then cap $\omega=+\infty$. If $\omega_{1} \subset \omega_{2}$, then $\mathscr{U}_{\omega 1} \supset \mathscr{U}_{\omega_{2}}$ so $\operatorname{cap} \omega_{1} \leqq \operatorname{cap} \omega_{2}$.

Definition 3.2. For open $\omega \subset X$, the set $P_{\omega} \subset F^{\prime}$ is the closure of the set of pure potentials $u^{f}$ where $f \geqq 0$ is a bounded measurable function with compact support contained in $\omega$, and with $\int f d \xi=1$.

It is immediate that $P_{\omega}$ is closed and convex.

Definition 3.3. For open $\omega \subset X$, the dual capacity of $\omega$ is

$$
\text { dualcap } \omega=\left\{\begin{array}{l}
\sup \left\{1 /\|z\| \mid z \in P_{\omega}\right\} \\
0 \text { for } P_{\omega}=\varnothing
\end{array} \quad \text { for } P_{\omega} \neq \varnothing\right.
$$


(Convention $1 / 0=+\infty$.)

REMARK. Definitions 3.1 and 3.3 are slightly different from their analogs used by Deny [4]. The change is required by technical problems due to the weaker assumptions. The change is not serious since it is clear that sets of zero exterior capacity are the same with either definition. Further, the exterior capacity herein is a true capacity in the sense of Brelot [2], (see $\S 4$ ).

LEMmA 3.4. Let $\left\{\omega_{i}\right\}_{i \in I}$ be a family of open subsets of $X$ directed upward by containment, with $\left\{\text { cap } \omega_{i}\right\}_{i \in I}$ a bounded set of real numbers. For each $i \in I$ denote by $u_{i} \in F$ the capacitary element associated with $\omega_{i}$. Then

(i) $\omega=\bigcup_{i \in I} \omega_{i}$ has a capacitary element $u$,

(ii) $u$ is the limit of the net $\left\{u_{i}\right\}_{i \in I}$.

Proof. By Lemma 1.3 with $E_{i}=\mathscr{U}_{\omega_{i}}, x_{i}=u_{i}$, and $x=u$, it follows that $\bigcap_{i \in I} \mathscr{U}_{\omega_{i}} \neq \varnothing$. Now $\mathscr{U}_{\omega}=\bigcap_{i \in I} \mathscr{U}_{\omega_{i}} \cdot$ In fact, $v \in \bigcap \mathscr{U}_{\omega_{i}}$ entails $v \geqq 1$ a.e. $\xi$ on $\omega_{i}$ for each $i \in I$, i.e., if $A_{i}=\left\{x \in \omega_{i} \mid v(x)<1\right\}$, then $\xi\left(A_{i}\right)=0$ for each $i \in I$. Let $A=\{x \in \omega \mid v(x)<1\}$, and compact

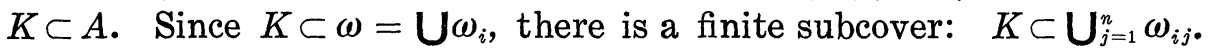
Since the family $\left\{\omega_{i}\right\}_{i \in I}$ is directed upward, there exists $i \in I$ with $K \subset \omega_{i}$, so $K \subset A_{i}$ and $\xi(K)=0$. Thus $\xi(A)=0$ and $\bigcap \mathscr{U}_{\omega_{i}} \subset \mathscr{U}_{\omega}$. The reverse containment is immediate. Lemma 1.3 gives the result.

In the proof of the following theorem it will be made clear that $\xi(\omega)=0$ entails cap $\omega=0$ for open $\omega$. Let $T: F^{\prime} \rightarrow F$ denote the duality map. Since $F$ is uniformly convex, $F^{\prime}$ is smooth so $T$ is unique.

THEOREM 3.5. For open $\omega \subset X$,

(i) dualcap $\omega=\operatorname{cap} \omega$,

(ii) if $0<$ dualcap $\omega<\infty$, the set

$$
E=\left\{v \in P_{\omega} \mid 1 /\|v\|=\text { dualcap } \omega\right\}
$$

is a nonvoid subset of $F^{\prime \prime}$. Moreover,

$$
T(E)=\|v\|^{2} \cdot u
$$

where $u \in F$ is the capacitary element associated with $\omega$.

Proof. Case 1. $\xi(\omega)=0$. Here $0 \in F$ is $\geqq 1$ a.e. $\xi$ on $\omega$ so cap $\omega=0$; any bounded measurable function $f$ supported by $\omega$ verifies $\int f d \xi=0$, so $P_{\omega}=\varnothing$, thus dualcap $\omega=0$. Hence $\operatorname{cap} \omega=$ dualcap $\omega$. 
In preparation for Cases 2 and 3, suppose $\xi(\omega)>0$. Let $K \subset \omega$ be compact with $\xi(K)>0$. Then $f=1 / \xi(K) \cdot I_{K}$ is an element of $P_{\omega}$, so $P_{\omega} \neq \varnothing$. Since $P_{\omega}$ is closed and convex and $F^{\prime \prime}$ is reflexive, it follows that $P_{\omega}$ has at least one element of minimum norm. Denote by $E$ the set of all such elements. Let $v \in E$ and consider $T(v) \in F$. For any $f: X \rightarrow R$ which generates a pure potential $u^{f} \in P_{\omega}$ we have $\left(T(v), u^{f}-v\right) \geqq 0$. In fact, if $v=0$, then $T v=0$ so $\left(T v, u^{f}-v\right)=0$. If $v \neq 0$,

$$
\begin{aligned}
& \frac{1}{\|v\|}\left(T v, u^{f}-v\right)=\lim _{t \rightarrow 0} \frac{\left\|v+t\left(u^{f}-v\right)\right\|-\|v\|}{t} \\
= & \lim _{t \rightarrow 0} \frac{\left\|(1-t) v+t u^{f}\right\|-\|v\|}{t} \geqq 0 .
\end{aligned}
$$

The limit exists by smoothness of $F^{\prime}$ and the inequality holds because $(1-t) v+t u^{f} \in P_{\omega}$ by convexity and the fact that $\|v\|$ is minimal over $P_{\omega}$. Thus for all such $u^{f} \in P_{\omega}$,

$$
\left(T v, u^{f}\right) \geqq(T v, v)=\|v\|^{2} .
$$

This inequality implies

$$
(T v)(x) \geqq\|v\|^{2} \text { a.e. } \xi \text { on } \omega .
$$

Case 2. $\xi(\omega)>0$ and $\operatorname{cap} \omega=+\infty$. This entails $\mathscr{C}_{\omega}=\varnothing$. Recall $v \in P_{\omega}$ and $1 /\|v\|=$ dualcap $\omega$. If $\|v\|^{2}>0$, then

$$
\left\{u \in F \mid u \geqq\|v\|^{2} \text { a.e. } \xi \text { on } \omega\right\}=\varnothing
$$

because $\mathscr{U}_{\omega}=\varnothing$. Hence (2) implies $\|v\|^{2}=0$, so $0=v \in P_{\omega}$. Thus, dualcap $\omega=+\infty$.

Case 3. $\xi(\omega)>0$ and $0<\operatorname{cap} \omega<+\infty$. Here $\mathscr{U}_{\omega} \neq \varnothing$. Let $u$ be the capacitary element associated with $\omega$. For any $u^{f} \in P_{\omega}$ we have $\int f d \xi=1$, then since $u \geqq 1$ a.e. $\xi$ on $\omega$,

$$
1 \leqq \int u f d \xi=\left(u, u^{f}\right) .
$$

But any $v \in P_{\omega}$ with $1 /\|v\|=$ dualcap $\omega$ is the limit of a sequence of such elements $u^{f}$, so

$$
1 \leqq(u, v) \leqq\|u\|\|v\|
$$

Thus, $\|v\| \neq 0$ and

$$
\frac{\|T v\|}{\|v\|} \leqq\|u\|\|v\| \frac{\|T v\|}{\|v\|^{2}}=\|u\| \cdot
$$


But $T v \geqq\|v\|^{2}$ a.e. on $\omega$ implies $T v /\|v\|^{2} \in \mathscr{C}_{\omega}$. Thus by the uniqueness of $u$ as the element of minimum norm in $\mathscr{Q}_{\omega}$, (3) implies $T v /\|v\|^{2}=u$, which verifies (ii). Finally,

$$
\operatorname{cap} \omega=\|u\|=\|T v\| /\|v\|^{2}=1 /\|v\|=\operatorname{dualcap} \omega
$$

which verifies (i).

4. Exterior capacity and capacitability.

DEFINITION 4.1. For any $E \subset X$, the exterior capacity of $E$ is defined by

$$
\operatorname{cap}_{e} E=\inf \{\operatorname{cap} \omega \mid \omega \supset E, \omega \text { open }\} .
$$

Observe that cap $_{e}$ is defined on all subsets of $X$, and that for $\omega$ open, $\operatorname{cap} \omega=\operatorname{cap}_{e} \omega$.

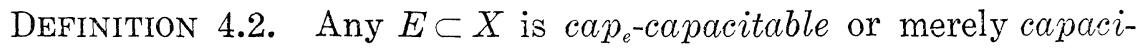
table, if

$$
\operatorname{cap}_{e} E=\sup \left\{\operatorname{cap}_{e} K \mid E \supset K, K \text { compact }\right\} \text {. }
$$

It will be shown that $\mathrm{cap}_{e}$ verifies

(i) cap $_{e}$ is increasing, i.e., $E_{1} \subset E_{2}$ implies cap $E_{1} \leqq$ cap $_{e} E_{2}$.

(ii) For any increasing sequence of $\operatorname{sets}\left\{E_{n}\right\}$,

$$
\lim _{n \rightarrow \infty} \operatorname{cap}_{e} E_{n}=\operatorname{cap}_{e} \bigcup_{n=1}^{\infty} E_{n} .
$$

(iii) For any decreasing sequence of compact sets $\left\{K_{n}\right\}$,

$$
\lim _{n \rightarrow \infty} \operatorname{cap}_{c} K_{n}=\operatorname{cap}_{e} \bigcap_{n=1}^{\infty} K_{n} \text {. }
$$

These are precisely the three conditions which must be verified in order that cap $_{e}$ be true capacity; it then follows that $K$-analytic subsets of $\sigma$-compact sets in $X$ are capacitable, see [2, Chapter I part II] and [3, Chapter VI]. In this section (i) and (iii) are indicated for $\operatorname{cap}_{e}$. That (ii) holds is shown in $\S 7$.

Proposition 4.3. The set function cap $_{e}$ verifies condition (i) for true capacity.

The proof follows immediately from the fact that cap is increasing on open sets.

DEFINITION 4.4. A set function $G$ is continuous on the right on 
compact sets if for any compact $K$ and $\varepsilon>0$ there is an open neighborhood $U \supset K$ such that $K^{\prime}$ compact and $K \subset K^{\prime} \subset U$ imply $G\left(K^{\prime}\right) \leqq$ $G(K)+\varepsilon$.

The notion "continuity on the right" is due to Choquet [3, pp. $147,174]$. The following lemma is from [2, p. 12].

LEMMA 4.5. Let $G$ be an increasing set function on the subsets of a Hausdorff space. If $G$ is continuous on the right on compact sets, then $G$ satisfies condition (iii) for true capacity.

Proposition 4.6. The set function cap $_{e}$ verifies condition (iii) for true capacity.

Proof. Let $K \subset X$ be compact and $\varepsilon>0$. By definition of cap there exists an open $\omega \supset K$ with $\operatorname{cap} \omega \leqq \operatorname{cap}_{e} K+\varepsilon$. Let a compact $K^{\prime}$ satisfy $K \subset K^{\prime} \subset \omega$. Then $\operatorname{cap}_{e} K^{\prime} \leqq \operatorname{cap} \omega \leqq \operatorname{cap}_{e} K+\varepsilon$. Lemma 4.5 now applies with $G=\operatorname{cap}_{e}$.

5. Some properties of cap $e_{e}$ Capacitability of open sets. The lemmas of this section lead to the proposition that open sets are capacitable. Moreover, the results of these lemmas are used in $\S 7$.

Definition 5.1. For any $E \subset X$, the set $\mathscr{C}_{E} \subset F$ is defined by

$$
\mathscr{U}_{E}=\left(\bigcup_{\omega \supset E} \mathscr{Q}_{\omega}\right)^{-}
$$

the union being over all open supersets of $E$. (Here the bar denotes closure.)

LeMma 5.2. (i) $\mathscr{U}_{E} \neq \varnothing$ iff $\operatorname{cap}_{e} E<\infty$.

(ii) For any $E \subset X, \mathscr{Q}_{E}$ is closed and convex.

(iii) In case $E=V$ is open, then $\mathscr{U}_{E}$ is identical to $\mathscr{U}_{V}$ of Definition 3.1.

Proof. (i) $\mathscr{U}_{E} \neq \varnothing$ iff for some open $\omega \supset E, \mathscr{U}_{\omega} \neq \varnothing$ iff for some open $\omega \supset E, \infty>\operatorname{cap} \omega$ iff $\infty>\operatorname{cap}_{e} E$.

(ii) Corollary 1.5 applies with $\left\{E_{i}\right\}_{i \in I}=\left\{\mathscr{U}_{\omega}\right\}_{\omega \supset E}$. Thus $\mathscr{L}_{E}$ is the closed convex hull of $\bigcup \mathscr{Q}_{\omega}$.

(iii) If $E=V$ is open, then $\mathscr{U}_{V} \supset \mathscr{U}_{\omega}$ for all open $\omega \supset V$. Thus

$$
\mathscr{U}_{V}=\mathscr{U}_{\bar{V}}^{-} \supset\left(\bigcup_{\omega \supset V} \mathscr{U}_{\omega}\right)^{-}=\left(\bigcup_{\omega \supset E} \mathscr{U}_{\omega}\right)^{-}=\mathscr{U}_{E} \cdot
$$

Conversely, $\mathscr{U}_{E} \supset \bigcup_{\omega \supset E} \mathscr{U}_{\omega} \supset \mathscr{U}_{V}$.

As a result of (ii) of the above lemma, we can give the following definition. 
Definition 5.3. For any $E \subset X$ with $\mathscr{U}_{E} \neq \varnothing$, the exterior capacitary element associated with $E, u_{E} \in F$, is the unique element of minimum norm of $\mathscr{L}_{E}$.

Lemma 5.4. Let $E \subset X$ with $\mathscr{Q}_{E} \neq \varnothing$. Then

(i) $\left\|u_{E}\right\|=\operatorname{cap}_{e} E$.

(ii) If $\left\{\omega_{i}\right\}_{i \in I}$ is any family of open sets in $X$ directed downward by inclusion with each $\omega_{i} \supset E$, $\operatorname{cap} \omega_{i}<\infty$ and $\operatorname{cap}_{e} E=\inf \left\{\operatorname{cap} \omega_{i} \mid i \in I\right\}$, then $u_{E}=\lim u_{i}$, where $u_{i}$ denotes the capacitary element associated with $\omega_{i}$.

Proof. (i ) Apply Corollary 1.5 with $\left\{E_{i}\right\}=\left\{\mathscr{U}_{\omega}\right\}, H=\mathscr{U}_{E}$.

(ii) Apply Lemma 1.4 with $K=\mathscr{U}_{E}$. By (i) above, $\|x\|=\left\|u_{E}\right\|=$ $\operatorname{cap}_{e} E$. By hypothesis,

$$
\operatorname{cap}_{e} E=\inf \left\{\operatorname{cap} \omega_{i} \mid i \in I\right\}=\inf \left\{\left\|x_{i}\right\| \mid i \in I\right\}
$$

in the notation of Lemma 1.4,

$$
u_{E}=x=\lim x_{i}=\lim u_{i} .
$$

Lemma 5.5. For any $E \subset X$ with $\operatorname{cap}_{e} E<\infty$, there exists a decreasing sequence of open sets $\left\{\omega_{n}\right\}_{n=1}^{\infty}$ with each $\omega_{n} \supset E$ and $u_{E}=$ $\lim u_{n}$, where $\left\{u_{n}\right\}_{n=1}^{\infty}$ is the corresponding sequence of capacitary elements.

Proof. From the family of all open supersets of $E$ with finite capacity, one uses an easy induction argument to construct a decreasing sequence $\left\{\omega_{n}\right\}$ with the property $\operatorname{cap}_{e} E=\lim$ cap $\omega_{n}$. The result follows by Lemma 5.4 .

For the purposes of the next corollary, we assume $\xi(E \cap K)=0$ for all compact $K$ implies $\xi E=0$.

Corollary 5.6. For $E \subset X$ with $\operatorname{cap}_{e} E<\infty, u_{E} \geqq 1$ a.e. $\xi$ on $E$. In case $\operatorname{cap}_{e} E=0$, then $\xi(E)=0$.

Proof. Let $\left\{\omega_{n}\right\},\left\{u_{n}\right\}$ be as in Lemma 5.5. Then $\lim u_{n}=u_{E}$. Let $K \subset E$ be compact. Axiom (a) assures $\lim _{n \rightarrow \infty} \int_{K}\left|u_{n}-u_{E}\right| d \xi=0$. Thus for some subsequence $u_{m}, \lim u_{m}=u_{E}$ pointwise a.e. $\xi$ on $K$. But $u_{m} \geqq 1$ a.e. $\xi$ on $\omega_{n}$, and hence a.e. $\xi$ on $K$. But $u_{m} \geqq 1$ a.e. $\xi$ on $\omega_{m}$, hence on $E \subset \omega_{m}$. Thus $u_{E}=\lim u_{m} \geqq 1$ a.e. $\xi$ on $E \cap K$, i.e., $u_{E} \geqq 1$ a.e. $\xi$ on $E$.

In case $\operatorname{cap}_{e} E=0$, we have $\left\|u_{E}\right\|=0$, so for any compact $K \subset X$, 


$$
0 \leqq \int_{E \cap K} u_{E} d \xi \leqq A \overline{(E \cap K)}\left\|u_{E}\right\|=0
$$

(here $A \overline{(E \cap K)}$ is the constant of axiom (a)). Thus, since $u_{E} \geqq 1$ a.e. $\xi$ on $E \cap K$, it follows that $\xi(E \cap K)=0$.

Proposition 5.7. Any open $\omega \subset X$ is capacitable.


$\sup \left\{\operatorname{cap}_{e} K \mid E \supset K\right.$ compact $\}=+\infty$, then $\operatorname{cap}_{e} E=+\infty$, so $E$ is capacitable. Thus it suffices to consider open $\omega$ satisfying $\sup \left\{\operatorname{cap}_{e} K \mid \omega \supset\right.$ $K$ compact $\}<\infty$. Using Lemmas 1.3 and 3.4 and Corollary 5.6, a proof similar to that given by Deny [4, pp 1-05, 1-06] for the Hilbert space case will suffice.

6. Denumerable sub-additivity of cap and $\mathrm{cap}_{e}$. In this section and the remainder of the article we assume that the uniformly convex space $F(X, \xi)$ satisfies axiom (c) as well as axiom(a). In this section the normal contraction "modulus", i.e., $u \rightarrow|u|$, is the only contraction needed, so the full strength of axiom (c) is not required.

Lemma 6.1. For any finite family of open subsets of $X$ we have

$$
\operatorname{cap} \bigcup_{i=1}^{n} \omega_{i} \leqq \sum_{i=1}^{n} \operatorname{cap} \omega_{i} \text {. }
$$

Proof. Without loss of generality, each $\mathscr{K}_{\omega_{i}} \neq \varnothing$. Let $u_{i} \in F$ be the capacitary element associated with $\omega_{i}, i=1, \cdots, n$. By axiom (c) $u \in \mathscr{U}_{\omega_{i}}$ implies $|u| \in \mathscr{U}_{\omega_{i}}$ and $\||u|\| \leqq\|u\|$. Thus $u_{i}=\left|u_{i}\right| \geqq 0$ a.e. $\xi$. Hence $\sum_{i=1}^{n} u_{i} \geqq 1$ a.e. $\xi$ on $\bigcup_{i=1}^{n} \omega_{i}$ so $\sum_{i}^{n} u_{i} \in \mathscr{U}_{\cup \omega_{i}}$, and

$$
\operatorname{cap} \mathbf{U}_{\omega_{i}} \leqq\left\|\sum u_{i}\right\| \leqq \sum\left\|u_{i}\right\|=\sum \operatorname{cap} \omega_{i} .
$$

REMARK. It is the last inequality in the above proof which makes our modified definition of capacity desirable.

Lemma 6.2. For any denumerable family of open subsets of $X$,

$$
\operatorname{cap} \bigcup_{i=1}^{\infty} \omega_{i} \leqq \sum_{i=1}^{\infty} \operatorname{cap} \omega_{i} \text {. }
$$

Proof. Assume $\mathscr{U}_{\omega_{i}} \neq \varnothing$. Put $0_{n}=\bigcup_{i=1}^{n} \omega_{n}, n=1,2, \cdots$. Then $\left\{0_{n}\right\}$ is strictly increasing. If $\lim \operatorname{cap} 0_{n}=\infty$, then

$$
\begin{aligned}
\infty & =\lim \operatorname{cap} 0_{n}=\lim _{n} \operatorname{cap} \bigcup_{i=1}^{n} \omega_{i} \\
& \leqq \lim _{n} \sum_{i=1}^{n} \operatorname{cap} \omega_{i}=\sum_{i=1}^{\infty} \operatorname{cap} \omega_{i},
\end{aligned}
$$


the inequality holds by Lemma 6.1. The result follows.

If lim cap $0_{n}<\infty$, the hypotheses of Lemma 3.4 are satisfied by $\left\{0_{n}\right\}$. Thus,

$$
\operatorname{cap} \bigcup_{i=1}^{\infty} \omega_{i}=\operatorname{cap} \bigcup_{n=1}^{\infty} 0_{n}=\lim \operatorname{cap} 0_{n}
$$

But

$$
\begin{gathered}
\lim \operatorname{cap} 0_{n}=\lim _{n} \operatorname{cap} \bigcup_{i=1}^{n} \omega_{i} \\
\leqq \\
\lim _{n} \sum_{i=1}^{n} \operatorname{cap} \omega_{i}=\sum_{i=1}^{\infty} \operatorname{cap} \omega_{i},
\end{gathered}
$$

the inequality holds by Lemma 6.1 .

Proposition 6.3. The set function cap $_{e}$ is denumerably subadditive, i.e., for a sequence of sets $\left\{E_{n}\right\}_{n=1}^{\infty}$

$$
\operatorname{cap}_{e} \bigcup_{n=1}^{\infty} E_{n} \leqq \sum_{n=1}^{\infty} \operatorname{cap}_{e} E_{n} \text {. }
$$

Proof. For each $n$, choose an open $\omega_{n} \supset E_{n}$ with cap $\omega_{n} \leqq$ $\operatorname{cap}_{e} E_{n}+\varepsilon / 2^{n}, \varepsilon>0$ preassigned. Then

$$
\operatorname{cap}_{e} \cup E_{n} \leqq \operatorname{cap} \bigcup \omega_{n} \leqq \sum \operatorname{cap} \omega_{n},
$$

the first inequality holds since cap $_{e}$ is increasing, the second by Lemma 6.2. By choice of $\omega_{n}, \sum \operatorname{cap} \omega_{n} \leqq \sum \operatorname{cap}_{e} E_{n}+\varepsilon$.

7. Quasi-continuous functions; exterior capacity is a true capacity. In this section definitions and results which lead to Theorem 7.12 are listed. Several proofs are omitted, but using the earlier results in this article, proofs similar to those in [4] can readily be supplied.

Definition 7.1. A function $f: X \rightarrow R$ is quasi-continuous if for each $\varepsilon>0$ there exists an open $\omega \subset X$ with cap $\omega<\varepsilon$ and the restriction $\left.f\right|_{x-\omega}$ is continuous.

DeFINITION 7.2. A statement is true quasi-everywhere (quasieverywhere on a subset $A \subset X)$ if it is true for all $x \in X-E(x \in A-E)$ and $\operatorname{cap}_{e} E=0$. The abbreviation is q.e. (q.e. on $A$ ).

By Corollary 5.6, q.e. implies a.e. $\xi$. It is emphasized that "q.e." depends not merely on the measure space $(X, \xi)$, but on the function space $F(X, \xi)$. Examples are given in $\S 8$.

Proposition 7.3. Let $f: X \rightarrow R$ be quasi-continuous, $V \subset X$ open, and $a \in R$ constant. Then $f \leqq a$ a.e. $\xi$ on $V$ implies $f \leqq a$ q.e. on $V$. 
The proof requires axioms (a) and (c), uniform convexity of $F$, and relies heavily on Theorem 3.5.

COROLLARY 7.4. Let $f, g$ be quasi-continuous functions. Then $f=g$ a.e. $\xi$ implies $f=g$ q.e. Consequently, since q.e. always implies a.e. $\xi, f$ and $g$ are quasi-continuous representatives of the same element $u \in F$ iff $f=g$ q.e.

Proof. Since $f=g$ a.e. $\xi$ implies $f-g \leqq 0$ a.e. $\xi$ and $g-f \leqq 0$ a.e. $\xi$, Proposition 7.3 gives $f-g \leqq 0$ q.e. and $g-f \leqq 0$ q.e., so $f=g$ q.e.

Definition 7.5. An element $u \in F$ is continuous if $u$ has a continuous representative.

LEMMA 7.6. Let $u$ denote any continuous representative of a continuous element of $F$ and $\|u\|$ the norm of that element of $F$. Then for any $a>0$,

$$
\operatorname{cap}\{x \mid u(x)>a>0\} \leqq\|u\| / a .
$$

Note. In [4], the right hand side of the above inequality is $\|u\|^{2} / a^{2}$, due to the different definition of capacity.

At this point axiom (b) is assumed. Consequently, from here on we will be concerned with a uniformly convex $B D$ space, $D(X, \xi)$. Observe that because axiom (b) requires $\xi$ to be dense in $X$, i.e., $\xi(\omega)>0$ for nonvoid open $\omega$, each continuous $u \in D$ has exactly one continuous representative, which is also denoted $u$.

Proposition 7.7. Every $u \in D$ has a quasi-continuous representative $(q=c$ rep $)$.

Proof. By axiom (b) there exists a sequence $\left\{u_{k}\right\}$ in $\mathscr{C} \cap D$ converging to $u$. By passing to a subsequence we may assume

$$
\sum_{k=1}^{\infty} 2^{k}\left\|u_{k+1}-u_{k}\right\|<+\infty \text {. }
$$

For each $k=1,2, \cdots$ put

$$
e_{k}=\left\{x|| u_{k+1}(x)-u_{k}(x) \mid>2^{-k}\right\} \text {. }
$$

By Lemma 7.6 and axiom (c)

$$
\text { cap } e_{k} \leqq 2^{k}\left\|\left|u_{k+1}-u_{k}\right|\right\| \leqq 2^{k}\left\|u_{k+1}-u_{k}\right\| \text {. }
$$

Put $\omega_{j}=\bigcup_{k=j}^{\infty} e_{k}$. Then $\left\{\omega_{j}\right\}_{j=1}^{\infty}$ is a decreasing sequence of open sets and by (4) 


$$
\operatorname{cap} \omega_{j} \leqq \sum_{k=j}^{\infty} 2^{k}\left\|u_{k+1}-u_{k}\right\| \longrightarrow 0
$$

as $j \rightarrow \infty ; \operatorname{cap}_{e}\left(\bigcap \omega_{j}\right)=0$ as a result, so $\xi\left(\bigcap \omega_{j}\right)=0$.

Clearly $\left\{u_{k}(x)\right\}$ is a convergent sequence of reals for $x \in X-\bigcap_{j=1}^{\infty} \omega_{j}$. Put

$$
u^{*}(x)=\left\{\begin{array}{l}
\lim u_{k}(x) \text { for } x \in X-\cap \omega_{j} \\
0 \text { for } x \in \bigcap \omega_{j}
\end{array}\right.
$$

The convergence $u_{k} \rightarrow u^{*}$ is uniform on the complement of any $\omega_{j}$, so $u^{*}$ is continuous there; thus $u^{*}$ is quasi-continuous since cap $\omega_{j} \rightarrow 0$.

Finally, if $u$ denotes any representative of $u \in D$, we show $u^{*}=u$ a.e. $\xi$. It suffices to show that for all $j, u^{*}=u$ a.e. $\xi$ on $X-\omega_{j}$. To this end, let $f$ be a bounded measurable function with compact $\mathscr{S}(f) \subset X-\omega_{j}$. Then

$$
\int u f d \xi=\lim _{k} \int u_{k} f d \xi=\int u^{*} f d \xi
$$

the first equality holds since $u_{k} \rightarrow u$ in $D$ and $\int(\cdot) f d \xi \in D^{\prime}$ by axiom (a); the second equality follows from the uniform convergence $u_{k} \rightarrow u^{*}$ on $X-\omega_{j}$. Thus $u=u^{*}$ a.e. $\xi$ on $X=\omega_{j}$ and $u^{*}$ is a $q=c$ rep of $u$.

LEMMA 7.8. If $u^{*}$ is any quasi-continuous representative of an element $u \in D$, then for any $a>0$,

$$
\operatorname{cap}_{e}\left\{x \mid u^{*}(x) \geqq a>0\right\} \leqq\|u\| / a \text {. }
$$

Proposition 7.9. Let $\left\{v_{n}\right\}$ be a sequence in $D$ converging to $v \in D$; let $v_{n}{ }^{*}, v^{*}$ be any quasi-continuous representatives of $v_{n}$, $v$ respectively. Then there exists a subsequence $\left\{{v_{n_{k}}}^{*}\right\}$ of $\left\{v_{n}{ }^{*}\right\}$ converging to $v^{*}$ quasi-everywhere.

LemMA 7.10. Let $E \subset X$ with $\operatorname{cap}_{e} E<+\infty$. If $u \in D$ has a quasi-continuous representative $u^{*} \geqq 1$ q.e. on $E$ and $u \geqq 0$ a.e. $\xi$ on $X$, then $u \in \mathscr{U}_{E}$ (Definition 5.1).

Proof. Let $u \in D$ satisfy the hypothesis, and $u^{*}$ be a $q=c$ rep of $u$. By adjusting $u^{*}$ on a set of exterior capacity zero and observing that $\lim _{n}(1+(1 / n)) u=u$ and $\mathscr{U}_{E}$ is closed, we see that the lemma will be proved if we assume $u^{*}>1$ everywhere on $E, \geqq 0$ a.e. $\xi$ on $X$, and show $u \in \mathscr{U}_{E}$.

Let $\varepsilon>0, \omega_{\varepsilon} \subset X$ open with cap $\omega_{\varepsilon}<\varepsilon$ and $\left.u^{*}\right|_{X-\omega_{\varepsilon}}$ continuous. Consider the open set 


$$
\Omega_{\varepsilon}=\left\{x \mid u^{*}(x)>1\right\} \bigcup \omega_{\varepsilon},
$$

and the capacitary element $v_{\varepsilon}$ associated with $\omega_{\varepsilon}$. Since $v_{\varepsilon} \geqq 1$ a.e. $\xi$ on $\omega_{\varepsilon}$ and by axiom (c) $v_{\varepsilon} \geqq 0$ a.e. $\xi$ on $X$, it follows that $u+v_{\varepsilon} \geqq 1$ a.e. $\xi$ on $\Omega_{\varepsilon}$, i.e., $u+v_{\varepsilon} \in \mathscr{U}_{\Omega_{\varepsilon}}$. But $\Omega_{\varepsilon} \supset E$ so $u+v_{\varepsilon} \in \mathscr{U}_{E}$. Letting $\varepsilon \rightarrow 0$ we have $\left\|v_{\varepsilon}\right\|=\operatorname{cap} \omega_{\varepsilon} \rightarrow 0$ so $u=\lim _{\varepsilon} u+v_{\varepsilon} \in \mathscr{U}_{E}$.

Lemma 7.11. Let $E \subset X$ with cap $_{e} E<\infty$. Then $u_{E}$, the exterior capacitary element associated with $E$, is $\geqq 0$ a.e. $\xi$ on $X$. Moreover, any quasi-continuous representative verifies $u_{E}{ }^{*} \geqq 1$ q.e. on $E$.

Proof. By axiom (c), the capacitary element associated with an open set is $\geqq 0$ a.e. $\xi$. Thus in the notation of Lemma 5.5, $\lim u_{n}=$ $u_{E}$ and $u_{n} \geqq 0$ a.e.g. Axiom (a) assures that the cone of nonnegative elements in $D$ is closed. Therefore, $u_{E} \geqq 0$ a.e. $\xi$.

Let $u_{E}{ }^{*}, u_{n}{ }^{*}$ be $q=c$ reps of $u_{E}, u_{n}$ respectively, $n=1,2, \cdots$. By Proposition $7.3, u_{n}{ }^{*} \geqq 1$ a.e. $\xi$ on $\omega_{n}$ implies $u_{n}{ }^{*} \geqq 1$ q.e. on $\omega_{n}$. Proposition 7.9 implies $u_{E}{ }^{*}(x)=\lim _{k} u_{n_{k}}{ }^{*}(x)$ q.e., so $u_{E}{ }^{*} \geqq 1$ q.e. on $\bigcap_{k=1}^{\infty} \omega_{n_{k}} \supset E$.

THEOREM 7.12. The set function $\mathrm{cap}_{e}$ is a true capacity.

Proof. In view of Propositions 4.3 and 4.6, it remains to show that $\mathrm{cap}_{e}$ verifies condition (ii) for a true capacity (see $\S 4$ ). Let a sequence $\left\{E_{n}\right\}$ of sets verify $E_{n} \subset E_{n+1}, n=1,2, \cdots$ and put $E=$ $\bigcup_{n=1}^{\infty} E_{n}$. Clearly $\operatorname{cap}_{e} E \geqq \lim _{n} \operatorname{cap}_{e} E_{n}$. We prove the reverse inequality.

If $\lim _{n} \operatorname{cap}_{e} E_{n}=+\infty$, equality holds. Assume cap $E_{n} \leqq M<+\infty$ for $n=1,2, \cdots$. Lemma 7.11 assures $u_{n} \geqq 0$ a.e. $\xi$ where $u_{n}$ denotes the exterior capacitary element associated with $E_{n}$. Put $\mathscr{U}=\bigcap_{n=1}^{\infty} \mathscr{U}_{E_{n}}$. Since $\left\{E_{n}\right\}$ is increasing, $\left\{\mathscr{U}_{E_{n}}\right\}$ is decreasing; further $\left\|u_{n}\right\|=\operatorname{cap}_{e} E_{n} \leqq$ $M$. Thus Lemma 1.3 applies: $u=\lim u_{n}$ is the unique element of minimum norm of $\mathscr{U}$. Observe that $u \geqq 0$ a.e. $\xi$ since $u_{n} \geqq 0$ a.e. $\xi$. If $u_{n}{ }^{*}$ is a $q=c$ rep of $u_{n}$, by applying Lemma 7.11 and adjusting $u_{n}{ }^{*}$ on a set of exterior capacity zero, we assume $u_{n}{ }^{*} \geqq 1$ everywhere on $E_{n}$. Proposition 7.9 assures $u^{*}=\lim _{k} u_{n_{k}}{ }^{*}$ q.e., so $u^{*} \geqq 1$ q.e. on $E=\bigcup_{k} E_{n_{k}}$. Thus by Lemma 7.10, $u \in \mathscr{U}_{E}$, so

$$
\operatorname{cap}_{e} E=\inf \left\{\|v\| \mid v \in \mathscr{U}_{E}\right\} \leqq\|u\|=\lim \operatorname{cap}_{e} E_{n} .
$$

8. Conditions under which $\xi(E)=0$ implies $\operatorname{cap}_{e}(E)=0$. In this section and the next we investigate the nature of sets of exterior capacity zero. In this section a connection is made with quasi-continuous functions; $\S 9$ deals with measures $\mu \in D^{\prime}$. The results of this section were motivated in part by the work of Thomas [7]; it is not 
hard to show that the union of the equivalence classes of a space $F(X, \xi)$ which is reflexive and satisfies axioms (a) and (c) forms a semi-norm space $\mathscr{E}(X, p)$ of [7].

To emphasize that "cap $E=0$ " depends on the functions in $D(X, \xi)$ and not merely on $\xi$, we give two brief examples of BD spaces over the same measure space but with diverse notions of capacity. In both examples $X=(0,1), \xi$ is Lebesgue measure, and $1<p<\infty$.

EXAMPLe 8.1. $D=L^{p}(X, \xi)$. We show that $\xi E=0$ implies $\operatorname{cap}_{e} E=0$ for $E \subset(0,1)$. In fact, given $\xi E=0$, cover $E$ with an open $\omega$ verifying $\xi \omega<\varepsilon, \varepsilon>0$ preassigned. The indicator $I_{\omega}$ is an $L^{p}$ function, and clearly

$$
\operatorname{cap} \omega=\left(\int I_{\omega}^{p} d \xi\right)^{1 / p}<\varepsilon^{1 / p} .
$$

Thus, $\operatorname{cap}_{e} E=\inf \{\operatorname{cap} \omega \mid \omega \supset E, \omega$ open $\}=0$.

EXAmple 8.2. $D(X, \xi)$ is the space of equivalence classes of $R$-valued functions on $(0,1)$, each class containing an absolutely continuous representative satisfying $\lim _{x \rightarrow 0} u(x)=\lim _{x \rightarrow 1} u(x)=0$. The norm is defined by

$$
\|u\|^{p}=\int\left|u^{\prime}\right|^{p} d \xi<\infty .
$$

Here the prime denotes derivative which may be taken in the ordinary sense in the case of the absolutely continuous representatives, or generally taken in the sense of distributions.

That $D$ is a BD space is an easy exercise. We show any open interval $\omega=(a, b)$ with $0<a<b<1$ verifies cap $\omega>2^{1 / p}$, from which it follows that $\operatorname{cap}_{e} E \geqq 2^{1 / p}$ for all nonvoid $E \subset(0,1)$. In fact, let $u \in \mathscr{U}_{\omega}$, i.e., $u \in D, u \geqq 1$ on $(a, b)$. (Here we are actually considering the absolutely continuous representative of $u$.) Then

$$
\|u\|^{p}=\int_{0}^{1}\left|u^{\prime}\right|^{p} d \xi \geqq \int_{0}^{a}\left|u^{\prime}\right|^{p} d \xi+\int_{b}^{1}\left|u^{\prime}\right|^{p} d \xi .
$$

By Hölder's inequality, $(q=p / p-1)$

$$
\left(\int_{0}^{a}\left|u^{\prime}\right|^{p} d \xi\right)^{1 / p} \geqq a^{-1 / q} \int_{0}^{a}\left|u^{\prime}\right| d \xi=a^{-1 / q} V_{0}^{a}(u) \geqq a^{-1 / q}>1 .
$$

Here the variation $V_{0}^{a}(u) \geqq 1$ since $u(\alpha) \geqq 1$ and $\lim _{x \rightarrow 0} u(x)=0$. Similarly $\int_{b}^{1}\left|u^{\prime}\right| d \xi>1$. Thus $\|u\|>2^{1 / p}$, so cap $\omega>2^{1 / p}$.

It is clear from this example that $\xi E=0$ does not generally imply $\operatorname{cap}_{e} E=0$. Conversely, Corollary 5.6 assures cap $e=0$ does 
entail $\xi E=0$. The next proposition gives one condition under which $\xi E=0$ and $\operatorname{cap}_{e} E=0$ are equivalent. We consider a uniformly convex $\mathrm{BD}$ space $D(X, \xi)$, and the indicator $I_{E}$ for $E \subset X$. (In Propositions 8.3 and 8.4 it is assumed that $\xi(E \cap K)=0$ for all compact $K$ implies $\xi E=0$; thus Corollary 5.6 applies.)

Proposition 8.3. If $I_{E}$ is quasi-continuous, then $\xi E=0$ iff $\operatorname{cap}_{e} E=0$.

Proof. Assume $I_{E}$ quasi-continuous and $\xi E=0$. Then $I_{E}$ is a $q=c$ rap of $0 \in D$. Since the null function is a $q=c$ rep of $0 \in D$, Corollary 7.4 assures $I_{E}=0$ q.e., so cap ca $_{e}=0$. Corollary 5.6 gives the converse.

As the following result indicates, in 8.1 all representatives of all elements in the space are quasi-continuous, but in 8.2 the only $q=c$ reps are the absolutely continuous representatives of each element.

Proposition 8.4. In order that for all $E \subset X \xi E=0$ implies $\operatorname{cap}_{e} E$, it is necessary and sufficient that all representatives of all elements of $D$ be quasi-continuous.

Proof. Necessity. Assume $\xi E=0$ implies $\operatorname{cap}_{e} E=0$. Let $u$ and $u^{*}$ be two representatives of the same element of $D, u^{*}$ quasicontinuous ( $u^{*}$ exists by Proposition 7.7). Put $E=\left\{x \mid u(x) \neq u^{*}(x)\right\}$. We have $\xi E=0$ so the hypothesis entails $\operatorname{cap}_{e} E=0$. Thus, $u=u^{*}$ q.e., so $u$ is quasi-continuous because $u^{*}$ is.

Sufficiency. If $\xi E=0$, then $I_{E}$ is a representative of $0 \in D$, so by hypothesis $I_{E}$ is quasi-continuous. Proposition 8.3 applies.

9. Sets of exterior capacity zero and pure potentials. It has been shown in previous sections that, roughly speaking, sets of zero capacity are smaller than sets of $\xi$-measure zero: cap $E=0$ implies $\xi E=0$. In this section we consider the question "how small is a set of zero exterior capacity?" More precisely, we give the following analog to a classical result: $\operatorname{cap}_{e} E=0$ iff $E$ is cap $_{e}$-capacitable and $\mu E=0$ for all pure potentials $u^{\mu}$. It is assumed $D(X, \xi)$ is a uniformly convex BD space.

For any open $\omega \subset X$, the characteristic function $I_{\omega}$ is lower semicontinuous. Consequently, for any Radon measure $\mu \geqq 0$, we have by definition

$$
\mu(\omega)=\mu^{*}\left(I_{\omega}\right)=\sup \left\{\mu(\varphi) \mid \varphi \in \mathscr{C}, \varnothing \leqq I_{\omega}\right\}
$$


The next lemma shows that the supremum can be taken over a smaller set. We use the normal contraction $T_{\varepsilon}: R \rightarrow R$ defined for $\varepsilon>0$ by $T_{\varepsilon}(x)=x-\varepsilon$ if $x \geqq \varepsilon, T_{\varepsilon}(x)=x+\varepsilon$ if $x \leqq-\varepsilon$ and $T(x)=0$ if $|x|<\varepsilon$.

Lemma 9.1. For any open $\omega \subset X$ and any Radon measure $\mu \geqq 0$

$$
\mu(\omega)=\sup \left\{\mu(\varphi) \mid \varphi \in \mathscr{C} \cap D, 0 \leqq \varphi \leqq I_{\omega}, \mathscr{S}(\varphi) \subset \omega\right\} .
$$

Proof. Let $\Gamma=\left\{\varphi \mid \varphi \in \mathscr{C} \cap D, 0 \leqq \varphi \leqq I_{\omega}, \mathscr{S}(\varphi) \subset \omega\right\}$. It suffices to show that $\Gamma$ is upward directed and $I_{\omega}=\sup \Gamma$ (see, for example, [5], Proposition 4.5.1). That $\Gamma$ is upward directed is immediate: $\varphi, \psi \in \Gamma$ implies $\varphi \vee \psi=1 / 2(\varphi+\psi+|\varphi-\psi|) \in \Gamma$ because $\mathscr{C} \cap D$ is a vector space closed under normal contractions (axiom (c)).

To see $I_{\omega}=\sup \Gamma$, let $p \in \omega, \psi \in \mathscr{C}$ with $\psi(p)=1,0 \leqq \psi \leqq 1$ on $X$ and $\mathscr{S}(\psi) \subset \omega$. Such a $\psi$ exists since $\{p\}$ is compact and $X$ is locally compact. Since $\mathscr{C} \cap D$ is dense in $\mathscr{C}$, given $\varepsilon, 0<\varepsilon<1 / 2$, there exists $\varphi \in \mathscr{C} \cap D$ with $|\varphi-\psi|<\varepsilon$ on $X$. An easy calculation shows $T_{\varepsilon} \mathscr{P} \in \Gamma$. Finally,

$$
\begin{gathered}
1-T_{\varepsilon} \varphi(p)=\psi(p)-T_{\varepsilon} \varphi(p) \\
=\psi(p)-\varphi(p)+\varepsilon<2 \varepsilon .
\end{gathered}
$$

Letting $\varepsilon$ tend to 0 ,

$$
I_{\omega}(p)=\sup \{\varphi(p) \mid \varphi \in \Gamma\},
$$

so $I_{\omega}=\sup \Gamma$.

For open $\omega \subset X$, recall $P_{\omega} \subset D^{\prime}$ given in Definition 3.2.

Lemma 9.2. For any open $\omega \subset X$ and $\mu \geqq 0$ for which $u^{\prime \prime} \in P_{\omega}$, $\mathscr{S}(\mu) \subset \bar{\omega}$ holds.

Proof. We show that any $p \in X-\bar{\omega}$ has a $\mu$-negligible neighborhood. Let $U$ be an open neighborhood of $p$ not meeting $\bar{\omega}$. Let $\varphi \in \mathscr{C} \cap D$ with $\varphi \leqq I_{U}, \mathscr{S}(\varphi) \subset U$. By definition of $u^{\mu} \in P_{\omega}$, there exists a sequence $f_{n}$ of bounded measurable functions supported by $\omega$ such that

$$
\int \varphi d \mu=\lim _{n} \int \varphi f_{n} d \xi=0
$$

Lemma 9.1 applied to $U$ gives $\mu(U)=0$.

THEOREM 9.3. Let $E \subset X$. The following two conditions are equivalent: 
(i) $E$ is cap $_{e}$-capacitable and $\mu E=0$ for every Radon measure $\mu \geqq 0$ generating a pure potential $u^{\mu} \in D^{\prime}$.

(ii) $\operatorname{cap}_{e} E=0$.

Proof. (i) implies (ii). First, we prove the contrapositive for compact $E$. Suppose $\operatorname{cap}_{e} E>0$, i.e., for some $\alpha>0$, cap $\omega \geqq \alpha$ for every open $\omega \supset E$. For every such $\omega$

$$
\alpha \leqq \text { dualcap } \omega=1 / \inf \left\{\|z\| z \in P_{\omega}\right\}
$$

and therefore $1 / \alpha \geqq \inf \left\{\|z\| \mid z \in P_{\omega}\right\}$. Now $P_{\omega}$ is convex and closed in $D^{\prime}$ and $\omega \subset \Omega$ implies $P_{\omega} \subset P_{\Omega}$ for open $\Omega$. Thus $\left\{P_{\omega} \mid \omega \supset E\right.$, $\omega$ open $\}$ satisfies the hypothesis of Lemma 1.3 (i) (here $D^{\prime}$ is reflexive because $D$ is uniformly convex), so there exists $z_{0} \in \cap \omega \supset E P_{\omega}$.

Now $z_{0} \neq 0$. In fact, $E$ is compact so some open $\omega \supset E$ is relatively compact. Therefore, since $z_{0} \in P_{\omega}$,

$$
+\infty>\operatorname{cap} \omega=\text { dualcap } \omega \geqq 1 /\left\|z_{0}\right\|,
$$

so $\left\|z_{0}\right\| \geqq 1 / \operatorname{cap} \omega>0$. Thus since $z_{0} \in P_{\omega}$, it follows that $z_{0}=u^{\mu}$ for some Radon measure $\mu>0$. We show $\mu E \geqq 1$. That $\mathscr{C} \cap D$ is dense in $\mathscr{C}$ assures the existence of $\varphi \in \mathscr{C} \cap D$ verifying $0 \leqq 1-\varphi<\varepsilon$ on the compact $\bar{\omega}$. Let $U \subset X$ be open with

$$
E \subset U \subset \bar{U} \subset \omega \text {. }
$$

Such $U$ exists because $E$ is compact and $X$ is locally compact Hausdorff. For all $u^{f} \in P_{\omega}$ with $0 \leqq f$ measurable, bounded and $\mathscr{S}(f) \subset U$, we have

$$
\int_{U} \varphi f d \xi \geqq(1-\varepsilon) \int_{U} f d \xi=1-\varepsilon,
$$

since $\varphi \geqq 1-\varepsilon$ on $\bar{\omega}$. By definition of $z_{0}=u^{\mu} \in P_{U}$, there exists a sequence $\left\{f_{n}\right\}$ of such functions so that

$$
1-\varepsilon \leqq \lim _{n} \int_{U} \varphi f_{n} d \xi=\int_{\bar{U}} \varphi d \mu
$$

by Lemma 9.2 , thus

$$
1-\varepsilon \leqq \int_{\omega} \varphi d \mu \leqq \int I_{\omega} d \mu=\mu(\omega) .
$$

Therefore,

$$
1-\varepsilon \leqq \inf \{\mu(\omega) \mid E \subset \omega \text { open }\}=\mu(E)
$$

so $\mu E \geqq 1$, and our result holds for compact sets $E$.

For the general case, $E$ capacitable means 


$$
\operatorname{cap}_{e} E=\sup \left\{\operatorname{cap}_{e} K \mid E \supset K \text { compact }\right\}=0
$$

since $\mu(E)=0$ assures $\mu(K)=0$ for $K \subset E$.

(ii) implies (i). For any open $\omega \subset X$ and any pure potential $u^{\mu}$, we establish the inequality

$$
\mu(\omega) \leqq\left\|u^{\prime \prime}\right\| \operatorname{cap} \omega
$$

In fact, by Lemma 9.1,

$$
\mu(\omega)=\sup \left\{\mu(\varphi) \mid \varphi \in \mathscr{C} \cap D, 0 \leqq \varphi \leqq I_{\omega}, \mathscr{S}(\varphi) \subset \omega\right\} .
$$

If $\operatorname{cap} \omega=+\infty$, the inequality holds. Assume cap $\omega<+\infty$, let $u \in D$ denote the capacitary element associated with $\omega$; then $I_{\omega} \leqq u$ a.e. $\xi$. Since $u^{\prime \prime}$ is a positive form on $D$, we have for any $\phi \in \Gamma$ (see Lemma 9.1),

$$
\begin{gathered}
\mu^{\prime}(\varphi)=\left(\varphi, u^{\prime \prime}\right) \leqq\left(u, u^{\prime \prime}\right) \\
\leqq u\|\| u^{\prime \prime}\|=\| u^{\mu} \| \operatorname{cap} \omega .
\end{gathered}
$$

Taking the supremum over all $\mu(\varphi), \varnothing \in \Gamma$, inequality (7) is proved.

Now, assume cap $\operatorname{cop}_{e} E=0$. Since cap $K=0$ for all compact $K \subset E$, it is immediate that $E$ is capacitable. By definition,

$$
0=\operatorname{cap}_{e} E=\inf \{\operatorname{cap} \omega \mid E \subset \omega \text { open }\} \text {. }
$$

By ( 7 )

$$
\begin{gathered}
\mu(E)=\inf \{\mu(\omega) \mid E \subset \omega \text { open }\} \\
\leqq \\
\left\|u^{\prime \prime}\right\| \inf \{\operatorname{cap} \omega \mid E \subset \omega \text { open }\}=0
\end{gathered}
$$

for all pure potentials $u^{\prime \prime}$.

10. Quasi-continuous representatives and pure potentials. In this section we indicate that by considering only the quasi-continuous representatives from each equivalence class $[u] \in D$, we get a "refined" space of equivalence classes of functions, the new equivalence relation being equality q.e. rather than equality a.e. $\xi$. An application of Theorems 9.3 and 10.1 give the important Corollary 10.2. Every representative in the "refined" space is measurable and summable with respect to any measure generating a pure potential and the "correct" integral formula holds. Our measure theoretic notation follows that of $[5, \S \S 4.5,4.6]$.

THEOREM 10.1. Every $u \in D$ has a quasi-continuous representative $u^{*}$ such that

(i) there exists some $\sigma$-compact subset of $X$ outside of which $u^{*}$ vanishes, and 
(ii) for every pure potential $u^{\mu} \in D^{\prime}$, we have $u^{*} \in \mathscr{L}^{1}(X, \mu)$ and

$$
\left(u, u^{\mu}\right)=\int u^{*} d \mu
$$

Proof. Refer to Proposition 7.7; we show that $u^{*}$ constructed in that proof verifies (i) and (ii). We have

$$
e_{k}=\left\{x \in X\left|u_{k+1}(x)-u_{k}(x)\right|>2^{-k}\right\}
$$

and

$$
\begin{gathered}
\mu\left(e_{k}\right) \leqq \mu\left(2^{k}\left|u_{k+1}-u_{k}\right|\right)=2^{k}\left(\left|u_{k+1}-u_{k}\right|, u^{\mu}\right) \\
\leqq 2^{k}\left\|\left|u_{k+1}-u_{k}\right|\right\| \cdot\left\|u^{\mu}\right\| \leqq 2^{k}\left\|u_{k+1}-u_{k}\right\| \cdot\left\|u^{\mu}\right\|,
\end{gathered}
$$

where $u^{\mu}$ is an arbitrary pure potential. The last quantity tends to zero as $k$ increases because

$$
\sum_{k=1}^{\infty} 2^{k}\left\|u_{k+1}-u_{k}\right\|<+\infty \text {. }
$$

Further, since $\omega_{j}=\bigcup_{k=j}^{\infty} e_{k}$, we have $\mu\left(\omega_{j}\right) \leqq \sum_{k=j}^{\infty} \mu\left(e_{k}\right)$ which tends to zero as $j$ increases by (8) and (9). Thus $\mu\left(\bigcap_{j=1}^{\infty} \omega_{j}\right)=0$. Also

$$
u^{*}(x)= \begin{cases}\lim _{k} u_{k}(x) & \text { for } x \in X-\cap \omega_{j} \\ 0 & \text { for } x \in \bigcap \omega_{j}\end{cases}
$$

Hence,

$$
\left\{x \mid u^{*}(x) \neq 0\right\} \subset \bigcup_{k=1}^{\infty}\left\{x \mid u_{k}(x) \neq 0\right\} \subset \bigcup_{k=1}^{\infty} \mathscr{S}\left(u_{k}\right)
$$

which establishes (i).

For (ii), let $E=\bigcap_{j=1}^{\infty} \omega_{j} ; \mu E=0$. Then

$$
\mu^{*}\left(\left|u^{*}-u_{k}\right|\right) \leqq \mu^{*}\left(\left|u^{*}-u_{k}\right| \cdot I_{X-E}\right)+\mu^{*}\left(\left|u^{*}-u_{k}\right| \cdot I_{E}\right)
$$

because the upper integral $\mu^{*}(\cdot)$ is sub-additive. But $\mu E=0$, so $\mu^{*}\left(\left|u^{*}-u_{k}\right| \cdot I_{E}\right)=0$. Thus

$$
\begin{aligned}
& \mu^{*}\left(\left|u^{*}-u_{k}\right|\right) \leqq \mu^{*}\left(\lim _{j}\left|u_{j}-u_{k}\right| \cdot I_{X-E}\right) \\
\leqq & \mu^{*}\left(\lim _{j}\left|u_{j}-u_{k}\right|\right) \leqq \underline{\lim }_{j} \mu^{*}\left(\left|u_{j}-u_{k}\right|\right)
\end{aligned}
$$

by Fatau's lemma. But $\left|u_{j}-u_{k}\right| \in \mathscr{C} \cap D$, so $\mu^{*}\left(\left|u_{j}-u_{k}\right|\right)=\mu\left(\mid u_{j}-\right.$ $\left.u_{k} \mid\right)$. Therefore,

$$
\begin{aligned}
& \mu^{*}\left(\left|u^{*}-u_{k}\right|\right) \leqq \lim _{j} \mu\left(u_{j}-u_{k}\right) \\
= & \underline{\lim }_{j}\left(\left|u_{j}-u_{k}\right|, u^{\mu}\right) \leqq \underline{\lim }_{j}\left\|u_{j}-u_{k}\right\| \cdot\left\|u^{\mu}\right\|
\end{aligned}
$$

which tends to zero as $k$ increases because $\left\{u_{k}\right\}$ is Cauchy in $D$. Thus $u^{*} \in \mathscr{L}^{1}(X, \mu)$ and 


$$
\int u^{*} d \mu=\lim _{k} \int u_{k} d \mu=\lim _{k}\left(u_{k}, u^{\mu}\right)=\left(u, u^{\mu}\right)
$$

COROLlaRY 10.2. Every quasi-continuous representative v of every $u \in D$ verifies $v \in \mathscr{L}^{1}(X, \mu)$ and $\left(u, u^{\prime \prime}\right)=\int v d \mu$ for every pure potential $u^{\mu}$.

Proof. Let $u^{*}$ be as in Theorem 10.1. Then $u^{*}=v$ a.e. $\xi$ and both are quasi-continuous. Thus Corollary 7.4 implies that $u^{*}=v$ q.e. Let

$$
E=\left\{x \in X \mid u^{*}(x) \neq v(x)\right\}
$$

then $\operatorname{cap}_{e} E=0$. According to Theorem 9.3, $\mu E=0$, so

$$
\int v d \mu=\int u^{*} d \mu=\left(u, u^{\prime \prime}\right)
$$

REMARK. The theorem and corollary give a very strong result. For an arbitrary representative $\tilde{u}$ of $u$, the formula

$$
\left(u, u^{\mu}\right)=\int \tilde{u} d \mu
$$

does not hold in general unless $\mu$ is absolutely continuous with respect to $\xi$. However, we can select any quasi-continuous representative $u^{*}$ of $u$ and the formula does hold, not just for one $\mu$, but for all $\mu$ simultaneously.

\section{REFERENCES}

1. A. Beurling and J. Deny, Dirichlet spaces, Proc. N. A. S., 45 (1959), 208-215.

2. M. Brelot, Lectures on Potential Theory, Tata Institute of Fundamental Research, Bombay, 1960.

3. G. Choquet, Theory of capacities, Ann. Institut Fourier, 5 (1955), 131-295.

4. J. Deny, Théorie de la capacité dans les espaces fonctionnels, Séminaire BrelotChoquet-Deny: Théorie du Potentiel t. 9 Faculté des Sciences de Paris, (1964-65), 1-01 to $1-13$.

5. R. E. Edwards, Functional Analysis-Theory and Applications, Holt, Rinehart, Winston, New York, 1965.

6. P. A. Fowler, Potential theory in Banach spaces of functions, a condensor theorem, J. Math. Anal. Appl., 33, 2 (1971), 310-322.

7. E. Thomas, Une axiomatique des espaces de Dirichlet, Séminaire Brelot-ChoquetDeny: Théorie du Potentiel t. 9 Faculté des Sciences de Paris, (1964-65), 9-01 to 9-04.

Received October 5, 1971 and in revised form April 26, 1973. Many of the results in this article are contained in the author's doctoral thesis which was directed by Professor J. Elliott, Rutgers University, New Brunswick, New Jersey, 1968. The work was supported in part by the National Science Foundation. 



\section{PACIFIC JOURNAL OF MATHEMATICS}

\section{EDITORS}

RICHARD ARENS (Managing Editor)

University of California

Los Angeles, California 90024

\author{
R. A. Beaumont \\ University of Washington \\ Seattle, Washington 98105
}

J. Dugundu*

Department of Mathematics

University of Southern California

Los Angeles, California 90007

D. Gilbarg and J. Milgram

Stanford University

Stanford, California 94305

\section{ASSOCIATE EDITORS}
E. F. BECKENBACH
B. H. NeumanN
F. WOLF
K. YosHIDA

\section{SUPPORTING INSTITUTIONS}

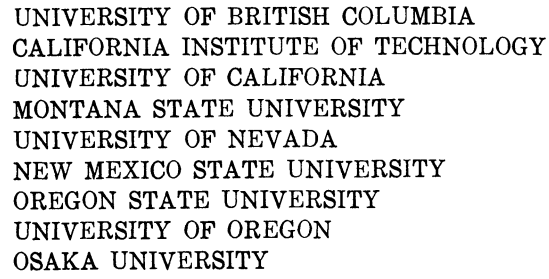

UNIVERSITY OF BRITISH COLUMBIA CALIFORNIA INSTITUTE OF TECHNOLOGY

UNIVERSITY OF CALIFORNIA

MONTANA STATE UNIVERSITY

UNIVERSITY OF NEVADA

NEW MEXICO STATE UNIVERSITY

OREGON STATE UNIVERSITY

UNIVERSITY OF OREGON

OSAKA UNIVERSITY

\author{
UNIVERSITY OF SOUTHERN CALIFORNIA \\ STANFORD UNIVERSITY \\ UNIVERSITY OF TOKYO \\ UNIVERSITY OF UTAH \\ WASHINGTON STATE UNIVERSITY \\ UNIVERSITY OF WASHINGTON \\ * * * \\ AMERICAN MATHEMATICAL SOCIETY \\ NAVAL WEAPONS CENTER
}

The Supporting Institutions listed above contribute to the cost of publication of this Journal, but they are not owners or publishers and have no responsibility for its content or policies.

Mathematical papers intended for publication in the Pacific Journal of Mathematics should be in typed form or offset-reproduced, (not dittoed), double spaced with large margins. Underline Greek letters in red, German in green, and script in blue. The first paragraph or two must be capable of being used separately as a synopsis of the entire paper. Items of the bibliography should not be cited there unless absolutely necessary, in which case they must be identified by author and Journal, rather than by item number. Manuscripts, in duplicate if possible, may be sent to any one of the four editors. Please classify according to the scheme of Math. Rev. Index to Vol. 39. All other communications to the editors should be addressed to the managing editor, or Elaine Barth, University of California, Los Angeles, California, 90024.

50 reprints are provided free for each article; additional copies may be obtained at cost in multiples of 50 .

The Pacific Journal of Mathematics is issued monthly as of January 1966. Regular subscription rate: $\$ 48.00$ a year (6 Vols., 12 issues). Special rate: $\$ 24.00$ a year to individual members of supporting institutions.

Subscriptions, orders for back numbers, and changes of address should be sent to Pacific Journal of Mathematics, 103 Highland Boulevard, Berkeley, California, 94708.

PUBLISHED BY PACIFIC JOURNAL OF MATHEMATICS, A NON-PROFIT CORPORATION

Printed at Kokusai Bunken Insatsusha (International Academic Printing Co., Ltd.), 270, 3-chome Totsuka-cho. Shinjuku-ku, Tokyo 160, Japan.

* C. R. DePrima California Institute of Technology, Pasadena, CA 91109, will replace J. Dugundji until August 1974.

Copyright (C) 1973 by

Pacific Journal of Mathematics

All Rights Reserved 


\section{Pacific Journal of Mathematics}

Vol. 48, No. $2 \quad$ April, 1973

Mir Maswood Ali, Content of the frustum of a simplex................

Mieczyslaw Altman, Contractors, approximate identities and factorization

in Banach algebras ................................ 323

Charles Francis Amelin, A numerical range for two linear operators ...... 335

John Robert Baxter and Rafael Van Severen Chacon, Nonlinear functionals



Stephen Dale Bronn, Cotorsion theories....................... 355

Peter A. Fowler, Capacity theory in Banach spaces............... 365

Jerome A. Goldstein, Groups of isometries on Orlicz spaces ........... 387

Kenneth R. Goodearl, Idealizers and nonsingular rings . ............ 395

Robert L. Griess, Jr., Automorphisms of extra special groups and

nonvanishing degree 2 cohomology ..................... 403

Paul M. Krajkiewicz, The Picard theorem for multianalytic functions . . . . 423

Peter A. McCoy, Value distribution of linear combinations of axisymmetric harmonic polynomials and their derivatives ...................

A. P. Morse and Donald Chesley Pfaff, Separative relations for

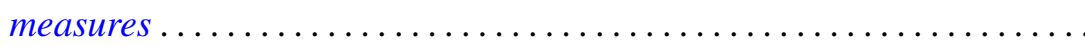

Albert David Polimeni, Groups in which $\operatorname{Aut}(G)$ is transitive on the

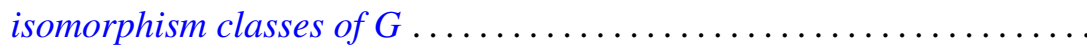

Aribindi Satyanarayan Rao, Matrix summability of a class of derived



Thomas Jay Sanders, Shape groups and products

Ruth Silverman, Decomposition of plane convex sets. II. Sets associated

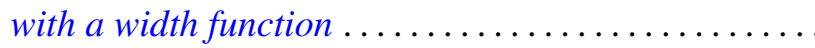

Richard Snay, Decompositions of $E^{3}$ into points and countably many flexible dendrites.............................

John Griggs Thompson, Nonsolvable finite groups all of whose local subgroups are solvable, IV ...

Robert E. Waterman, Invariant subspaces, similarity and isometric equivalence of certain commuting operators in $L_{p} \ldots$

James Chin-Sze Wong, An ergodic property of locally compact amenable

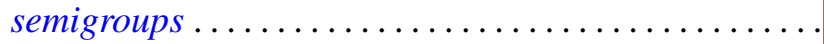

Julius Martin Zelmanowitz, Orders in simple Artinian rings are strongly equivalent to matrix rings ....................... 